\title{
Plus-one generated and next to free arrangements of hyperplanes
}

\author{
Takuro Abe *
}

August 20, 2018

\begin{abstract}
We introduce a new class of arrangements of hyperplanes, called (strictly) plus-one generated arrangements, from algebraic point of view. Plus-one generatedness is close to freeness, i.e., plus-one generated arrangements have their logarithmic derivation modules generated by dimension plus one elements, with relations containing one linear form coefficient. We show that strictly plus-one generated arrangements can be obtained if we delete a hyperplane from free arrangements. We show a relative freeness criterion in terms of plus-one generatedness. In particular, for plane arrangements, we show that a free arrangement is in fact surrounded by free or strictly plus-one generated arrangements. We also give several applications.
\end{abstract}

\section{Introduction}

To state the main results, let us introduce a part of notations. See $\S 2$ for details. Let $\mathbb{K}$ be a field, $V=\mathbb{K}^{\ell}$ and $S=\operatorname{Sym}^{*}\left(V^{*}\right)$ the coordinate ring. Let $x_{1}, \ldots, x_{\ell}$ be a basis for $V^{*}$ so that $S=\mathbb{K}\left[x_{1}, \ldots, x_{\ell}\right]$, and that Der $S=$ $\bigoplus_{i=1}^{\ell} S \partial_{x_{i}}$. An arrangement of hyperplanes $\mathcal{A}$ in $V$ is a finite set of linear hyperplanes in $V$. We assume that every $\mathcal{A}$ is essential unless otherwise specified, i.e., $\cap_{H \in \mathcal{A}} H=\{0\}$. For $H \in \mathcal{A}$, let

$$
\mathcal{A}^{H}:=\{L \cap H \mid L \in \mathcal{A} \backslash\{H\}\}
$$

\footnotetext{
*Institute of Mathematics for Industry, Kyushu University, Fukuoka 819-0395, Japan. Email:abe@imi.kyushu-u.ac.jp. Tel:+81-92-802-4479. 2010 Mathematics Subject Classification. 32S22, 52S35.
} 
be the restriction of $\mathcal{A}$ onto $H$, which is an arrangement in $H$. For each $H \in$ $\mathcal{A}$ fix a linear form $\alpha_{H} \in V^{*}$ such that $\operatorname{ker} \alpha_{H}=H$. Then the logarithmic derivation module $D(\mathcal{A})$ of $\mathcal{A}$ is defined by

$$
D(\mathcal{A}):=\left\{\theta \in \operatorname{Der} S \mid \theta\left(\alpha_{H}\right) \in S \alpha_{H}(\forall H \in \mathcal{A})\right\} .
$$

In general, $D(\mathcal{A})$ is a reflexive graded $S$-module, and not free. So we say that $\mathcal{A}$ is free with exponents $\exp (\mathcal{A})=\left(d_{1}, \ldots, d_{\ell}\right)$ if there exist homogeneous derivations $\theta_{1}, \ldots, \theta_{\ell} \in D(\mathcal{A})$ such that $D(\mathcal{A})=\bigoplus_{i=1}^{\ell} S \theta_{i}$ and that $\operatorname{deg} \theta_{i}=$ $d_{i}(i=1, \ldots, \ell)$. Here $\theta=\sum_{i=1}^{\ell} f_{i} \partial_{x_{i}} \in \operatorname{Der} S$ is homogeneous of degree $d$ if all $f_{i}$ belongs to the homogeneous degree $d$-part $S_{d}$ of $S$.

Free arrangements have been intensively studied as they are the most important algebraic properties of arrangements. However, only few of algebraic structures of $D(\mathcal{A})$ is known when $\mathcal{A}$ is not free. In general, it is almost impossible to determine the algebraic structure of a non-free $D(\mathcal{A})$ without using a computer program. In this article, we introduce a new class of arrangements from the algebraic point of view, which are next easiest to free arrangements as follows:

Definition 1.1

(1) We say that $\mathcal{A}$ is plus-one generated with exponents $\operatorname{PO} \exp (\mathcal{A})=$ $\left(d_{1}, \ldots, d_{\ell}\right)$ and level $d$ if $D(\mathcal{A})$ has a minimal free resolution of the following form:

$$
0 \rightarrow S[-d-1] \stackrel{\left(\alpha, f_{1}, \ldots, f_{\ell}\right)}{\longrightarrow} S[-d] \oplus\left(\oplus_{i=1}^{\ell} S\left[-d_{i}\right]\right) \rightarrow D(\mathcal{A}) \rightarrow 0 .
$$

(2) We say that $\mathcal{A}$ is strictly plus-one generated with exponents $\operatorname{PO} \exp (\mathcal{A})=\left(d_{1}, \ldots, d_{\ell}\right)$ and level $d$ if $\mathcal{A}$ is plus-one generated with the same exponents and level, and $\alpha \neq 0$ in the resolution (1.1).

\section{Remark 1.2}

(1) In other words, $\mathcal{A}$ is plus-one generated if there is a minimal set of homogeneous generators $\theta_{E}=\theta_{1}, \theta_{2}, \ldots, \theta_{\ell}$ and $\varphi$ for $D(\mathcal{A})$ such that $\operatorname{deg} \theta_{i}=$ $d_{i}, \operatorname{deg} \varphi=d$ and

$$
\sum_{i=1}^{\ell} f_{i} \theta_{i}+\alpha \varphi=0,
$$

where $f_{i} \in S, \alpha \in V^{*}$. This $\alpha$ is called the level coefficient, and $\varphi$ a level element. If it is strict, then $\alpha \neq 0$.

(2) When $d=d_{i}$ for some $i$, then the choice of the level coefficient and element is not unique. Hence the strict plus-one generatedness means that there is a choice of generators satisfying the conditions in Definiton 1.1 (2). 
(3) Recall that for all $H \in \mathcal{A}$, there is a decomposition

$$
D(\mathcal{A}) \simeq S \theta_{E} \oplus D_{H}(\mathcal{A})
$$

where $\theta_{E}$ is the Euler vector field and $D_{H}(\mathcal{A}):=\left\{\theta \in D(\mathcal{A}) \mid \theta\left(\alpha_{H}\right)=\right.$ 0\}. See Lemma 1.33 in [23] for example. Hence choosing the generators appropriately, we may assume that the relation (1.1) exists in $D_{H}(\mathcal{A})$.

(4) By definition, for a plus-one generated arrangement $\mathcal{A}$, its exponents and level cannot be computed by its characteristic polynomial. Conversely, we can compute the first and second Betti numbers. See Proposition 4.1.

As the definition says, plus-one generated arrangements are not free, and have simple algebraic structures, which is very close to that of free arrangements. If $d=\max _{i}\left\{d_{i}\right\}$ and $\ell=3,4$, then it coincides with the definition of nearly free line and plane arrangements defined by Dimca and Sticularu in $[12$ and [13]. Definition 1.1 is motivated by them, and gives a generalization of them. On the other hand, we can define the arrangement close to the free arrangement in the sense of the inclusion relation as follows:

\section{Definition 1.3}

(1) We say that $\mathcal{A}$ is next to free minus (NT-free minus) if there is a free arrangement $\mathcal{B}$ and $H \in \mathcal{B}$ such that $\mathcal{A}=\mathcal{B} \backslash\{H\}$.

(2) We say that $\mathcal{A}$ is next to free plus (NT-free plus) if there is a free arrangement $\mathcal{B}$ and a hyperplane $H \notin \mathcal{B}$ such that $\mathcal{A}=\mathcal{B} \cup\{H\}$.

(3) $\mathcal{A}$ is next to free if $\mathcal{A}$ is either next to free plus or minus.

Since both arrangements above are close to free arrangements in different sense, it is natural to ask whether they are related. In fact, we can show that next to free minus arrangements are free, or strictly plus-one generated:

\section{Theorem 1.4}

Let $\mathcal{A}$ be free with $\exp (\mathcal{A})=\left(d_{1}, \ldots, d_{\ell}\right)$ and $H \in \mathcal{A}$. Then $\mathcal{A}^{\prime}:=\mathcal{A} \backslash\{H\}$ is free, or strictly plus-one generated with exponents $\left(d_{1}, \ldots, d_{\ell}\right)$ and level $d=\left|\mathcal{A}^{\prime}\right|-\left|\mathcal{A}^{H}\right|$.

Theorem 1.4 says that, if an arrangement is close to the free arrangement in the sense of the inclusion relation, then so is as an algebraic structure. As an example, we give an arrangement which is not free but whose algebraic structure can be determined by Theorem 1.4 without any algebraic computation. 


\section{Example 1.5}

Let $\ell \geq 2$ and $\mathcal{A}$ be an arrangement in $\mathbb{R}^{\ell+1}$ defined by

$Q(\mathcal{A})=z \prod_{i=1}^{\ell} x_{i} \prod_{i=1}^{\ell}\left(x_{i}-z\right) \prod_{1 \leq i<j \leq \ell}\left(x_{i}^{2}-x_{j}^{2}\right) \prod_{1 \leq i<j \leq \ell}\left(x_{i}-x_{j}-z\right) \prod_{1 \leq i<j \leq \ell}\left(x_{i}+x_{j}-z\right)$.

This is the Shi arrangement of the type $B_{\ell}$, which is free with $\exp (\mathcal{A})=$ $\left(1,(2 \ell)^{\ell}\right)$ by [21]. Here $(a)^{b}$ stands for the $b$-copies of a for nonnegative integers $a, b$. Let $H=\{z=0\}$ and $\mathcal{A}^{\prime}:=\mathcal{A} \backslash\{H\}$. Then $\left|\mathcal{A}^{H}\right|=\ell^{2}$. Thus $|\mathcal{A}|-\left|\mathcal{A}^{H}\right|=\ell^{2}+1 \notin \exp (\mathcal{A})$, and $\mathcal{A}^{\prime}$ is not free. By Theorem 1.4, $\mathcal{A}$ is strictly plus-one generated with $\operatorname{PO} \exp \left(\mathcal{A}^{\prime}\right)=\left(1,(2 \ell)^{\ell}\right)$ and level $\ell^{2}$, i.e.,

$$
0 \rightarrow S\left[-\ell^{2}-1\right] \rightarrow S\left[-\ell^{2}\right] \oplus S[-1] \oplus S[-2 \ell]^{\oplus \ell} \rightarrow D\left(\mathcal{A}^{\prime}\right) \rightarrow 0 .
$$

An immediate corollary of Theorem 1.4 is as follows.

\section{Corollary 1.6}

Let $\mathcal{A}$ be free, $\mathcal{A} \ni H$ and $\mathcal{A}^{\prime}:=\mathcal{A} \backslash\{H\}$. Then

(1) the projective dimension $\operatorname{pd}_{S} D\left(\mathcal{A}^{\prime}\right) \leq 1$, and

(2) $D\left(\mathcal{A}^{\prime}\right)$ is generated by at most $(\ell+1)$-derivations.

A corollary of Theorem 1.4 is the following criterion for freeness when the level is strictly higher than exponents, which is relative compared with other criterions like by K. Saito in [17], or Yoshinaga in [21], [22], [10].

\section{Theorem 1.7 (Relative freeness criterion)}

Let $H \in \mathcal{A}, \mathcal{A}^{\prime}:=\mathcal{A} \backslash\{H\}$, and let $\left|\mathcal{A}^{\prime}\right|-\left|\mathcal{A}^{H}\right|=: d$. Then $\mathcal{A}$ is free if and only if either

(1) $\mathcal{A}^{H}$ is free and $\chi\left(\mathcal{A}^{H} ; t\right) \mid \chi(\mathcal{A} ; t)$, or

(2) $\mathcal{A}^{\prime}$ is strictly plus-one generated with level element $\varphi \in D\left(\mathcal{A}^{\prime}\right)_{d}$, and $D\left(\mathcal{A}^{\prime}\right)_{d} \backslash \mathbb{K} \varphi \subset D(\mathcal{A})_{d}$.

In case $(1), \exp (\mathcal{A}) \cap \exp \left(\mathcal{A}^{\prime}\right)=\exp \left(\mathcal{A}^{H}\right)$, and in case $(2), \exp (\mathcal{A})=\operatorname{POexp}\left(\mathcal{A}^{\prime}\right)$.

Theorem 1.7 (2) requires very strong conditions which is hard to check. However, this is the first criterion of freeness in which we use the information on the triple $\left(\mathcal{A}, \mathcal{A}^{\prime}, \mathcal{A}^{H}\right)$. Thus if we can improve (2), then we may apply Theorems 2.4 or 2.5 to approach Terao's conjecture. If the level is strictly larger than exponents, then the criterion becomes simpler. 
Theorem 1.8 (Relative freeness criterion with large level)

Let $H \in \mathcal{A}, \mathcal{A}^{\prime}:=\mathcal{A} \backslash\{H\}$. Assume that $\chi(\mathcal{A} ; t)=\prod_{i=1}^{\ell}\left(t-d_{i}\right)$, and $\left|\mathcal{A}^{\prime}\right|-\left|\mathcal{A}^{H}\right|=: d>d_{i}(\forall i)$. Then $\mathcal{A}$ is free if and only if $\mathcal{A}^{\prime}$ is strictly plus-one generated with $\operatorname{PO} \exp (\mathcal{A})=\left(d_{1}, \ldots, d_{\ell}\right)$. In this case, $\exp (\mathcal{A})=\operatorname{POexp}\left(\mathcal{A}^{\prime}\right)$ and the level is $d$.

We have the addition version of Theorem 1.4 as follows:

\section{Theorem 1.9}

Let $\mathcal{A}$ be an arrangement, $H \in \mathcal{A}$ and $\mathcal{A}^{\prime}:=\mathcal{A} \backslash\{H\}$. Assume that $\mathcal{A}^{\prime}$ is free with $\exp \left(\mathcal{A}^{\prime}\right)=\left(d_{1}, \ldots, d_{\ell}\right)_{\leq}$. If $d:=\left|\mathcal{A}^{\prime}\right|-\left|\mathcal{A}^{H}\right| \geq d_{\ell-2}$, then $\mathcal{A}$ is free, or strictly plus-one generated with $\operatorname{PO} \exp (\mathcal{A})=\left(d_{1}, \ldots, d_{\ell-2}, d_{\ell-1}+1, d_{\ell}+1\right)$ and level $d_{\ell-1}+d_{\ell}-|\mathcal{A}|+\left|\mathcal{A}^{H}\right|+1$.

Here $\left(d_{1}, \ldots, d_{\ell}\right)_{\leq}$stands for $d_{1} \leq \cdots \leq d_{\ell}$.

\section{Remark 1.10}

In fact, Theorem 1.9 can be divided into two statements, i.e., $\mathcal{A}$ is free if $d_{\ell-1} \leq d \leq d_{\ell}$, and free or strictly plus-one generated when $d_{\ell-2} \leq d<d_{\ell-1}$. The former result is due to Hoge in [15]. See Theorem 2.13 for details.

As we can see, Theorems 1.4 and 1.9 are not symmetric. In fact, the addition does not work well as the deletion as in Example 7.5. When $\ell=$ 3, contrary to Example 7.5, the complete symmetry exists, which gives a complete relative criterion for the freeness.

Theorem 1.11 (Relative freeness criterion for plane arrangements) Let $\ell=3$. Then the following are equivalent.

(1) $\mathcal{A}$ is free with $\exp (\mathcal{A})=(1, a, b)$.

(2) For any $H \in \mathcal{A}, \mathcal{A}^{\prime}:=\mathcal{A} \backslash\{H\}$ is either

(i) free with $\exp \left(\mathcal{A}^{\prime}\right)=(1, a, b-1)$ and $\left|\mathcal{A}^{\prime}\right|-\left|\mathcal{A}^{H}\right|=b-1$, or

(ii) (strictly) plus-one generated with $\operatorname{PO} \exp \left(\mathcal{A}^{\prime}\right)=(1, a, b)$ and level $d=\left|\mathcal{A}^{\prime}\right|-\left|\mathcal{A}^{H}\right|$.

(3) For some $H \in \mathcal{A}, \mathcal{A}^{\prime}:=\mathcal{A} \backslash\{H\}$ is either

(i) free with $\exp \left(\mathcal{A}^{\prime}\right)=(1, a, b-1)$ and $\left|\mathcal{A}^{\prime}\right|-\left|\mathcal{A}^{H}\right|=b-1$, or

(ii) (strictly) plus-one generated with $\operatorname{PO} \exp \left(\mathcal{A}^{\prime}\right)=(1, a, b)$ and level $d=\left|\mathcal{A}^{\prime}\right|-\left|\mathcal{A}^{H}\right|$. 
(4) For any plane $L \notin \mathcal{A}, \mathcal{B}:=\mathcal{A} \cup\{L\}$ is either

(i) free with $\exp (\mathcal{B})=(1, a, b+1)$ and $\left|\mathcal{B}^{H}\right|=a+1$, or

(ii) (strictly) plus-one generated with $\operatorname{PO} \exp (\mathcal{B})=(1, a+1, b+1)$ and level $d=\left|\mathcal{B}^{H}\right|-1$.

(5) For some plane $L \notin \mathcal{A}, \mathcal{B}:=\mathcal{A} \cup\{L\}$ is either

(i) free with $\exp (\mathcal{B})=(1, a, b+1)$ and $\left|\mathcal{B}^{H}\right|=a+1$, or

(ii) (strictly) plus-one generated with $\operatorname{PO} \exp (\mathcal{B})=(1, a+1, b+1)$ and level $d=\left|\mathcal{B}^{H}\right|-1$.

\section{Remark 1.12}

When $\ell=3$, plus-one generatedness coincides with strictly plus-one generatedness. See Proposition 5.1.

As Theorem 1.11 implies, for plane arrangements, next to free arrangements are free or strictly plus-one generated. Also, free arrangements are surrounded by free or strictly plus-one generated arrangements.

We say that $\mathcal{A}$ has a free filtration

$$
\emptyset=\mathcal{A}_{0} \subset \mathcal{A}_{1} \subset \cdots \subset \mathcal{A}_{n}=\mathcal{A}
$$

if $\left|\mathcal{A}_{i}\right|=i$ and $\mathcal{A}_{i}$ is free for $i=0, \ldots, n$. It is shown in [4] that whether a free arrangement has a free filtration or not depends only on combinatorics. By using plus-one generatedness, we can show the following:

\section{Theorem 1.13}

Let $\ell \leq 4$. Then whether $\mathcal{A}$ has a free filtration depends only on $L(\mathcal{A})$.

The organization of this article is as follows. $§ 2$ is devoted for recalling previous results and definitions. In $\S 3$, we introduce two tricks which are often used in this article, the replacement and locally free tricks. In $\S 4$ we prove the main results except for Theorem 1.11, which will be proved in $\S 5$. In $\S 6$ we consider whether a given non-free arrangement can admit an addition which makes it free. In $\S 7$ we introduce a lot of interesting examples on free, next to free and plus-one generated arrangements. In $\S 8$ we consider another expression of the combinatoriality of the deletion theorem first proved in [4].

Acknowledgements. The author is grateful to M. Barakat, M. Cuntz, T. Hoge and $\mathrm{H}$. Terao for the permission to put Theorem 2.13 in this article. Also, the author is grateful to Anca Macnic for a comment to this article. The author is partially supported by JSPS Grant-in-Aid for Scientific Research (B) JP16H03924, and Grant-in-Aid for Exploratory Research JP16K13744. 


\section{Preliminaries}

In this section let us summarize several results and definitions. Start from combinatorics and topology of arrangements. Let

$$
L(\mathcal{A}):=\left\{\bigcap_{H \in \mathcal{B}} H \mid \mathcal{B} \subset \mathcal{A}\right\}
$$

be the intersection lattice of $\mathcal{A}$ with a partial order induced from the reverse inclusion. Define $L_{i}(\mathcal{A}):=\left\{X \in L(\mathcal{A}) \mid \operatorname{codim}_{V} X=i\right\}$. The Möbius function $\mu: L(\mathcal{A}) \rightarrow \mathbb{Z}$ is defined by $\mu(V)=1$, and by

$$
\mu(X):=-\sum_{Y \in L(\mathcal{A}), X \subsetneq Y \subset V} \mu(Y)
$$

for $X \in L(\mathcal{A}) \backslash\{V\}$. Define the characteristic polynomial $\chi(\mathcal{A} ; t)$ by

$$
\chi(\mathcal{A} ; t):=\sum_{X \in L(\mathcal{A})} \mu(X) t^{\operatorname{dim} X},
$$

and the Poincaré polynomial $\pi(\mathcal{A} ; t)$ by

$$
\pi(\mathcal{A} ; t):=\sum_{X \in L(\mathcal{A})} \mu(X)(-t)^{\operatorname{codim} X} .
$$

It is clear that

$$
\pi(\mathcal{A} ; t)=(-t)^{\ell} \chi\left(\mathcal{A} ;-t^{-1}\right)
$$

For $X \in L(\mathcal{A})$, the localization $\mathcal{A}_{X}$ of $\mathcal{A}$ at $X$ is defined by

$$
\mathcal{A}_{X}:=\{H \in \mathcal{A} \mid X \subset H\},
$$

and the restriction $\mathcal{A}^{X}$ of $\mathcal{A}$ onto $X$ is defined by

$$
\mathcal{A}^{X}:=\left\{H \cap X \mid H \in \mathcal{A} \backslash \mathcal{A}_{X}\right\} .
$$

We agree that, for $H \in \mathcal{A}$ and $X \in L\left(\mathcal{A}^{H}\right), \mathcal{A}_{X}^{H}$ stands for $\left(\mathcal{A}_{X}\right)^{H}$. It is easy to show that $\mathcal{A}_{X}^{H}=\left(\mathcal{A}^{H}\right)_{X}$ too.

The characteristic polynomial $\chi(\mathcal{A} ; t)$ is combinatorial but not easy to compute in general. The most useful inductive method to compute $\chi(\mathcal{A} ; t)$ is so called the deletion-restriction formula (e.g., Corollary 2.57 in [16]) as follows:

$$
\chi(\mathcal{A} ; t)=\chi(\mathcal{A} \backslash\{H\} ; t)-\chi\left(\mathcal{A}^{H} ; t\right) .
$$


Let $\chi(\mathcal{A} ; t)=\sum_{i=0}^{\ell}(-1)^{i} b_{i}(\mathcal{A}) t^{\ell-i}$. When $\mathcal{A} \neq \emptyset$, it is known that $\chi(\mathcal{A} ; t)$ is divisible by $t-1$. Define $\chi_{0}(\mathcal{A} ; t):=\chi(\mathcal{A} ; t) /(t-1):=\sum_{i=0}^{\ell-1}(-1)^{i} b_{i}^{0}(\mathcal{A}) t^{\ell-i-1}$. By the definition, $b_{1}(\mathcal{A})=|\mathcal{A}|, b_{1}^{0}(A)=|\mathcal{A}|-1$ and

$$
b_{2}^{0}(\mathcal{A})=b_{2}(\mathcal{A})-(|\mathcal{A}|-1)
$$

It is known that $b_{i}(\mathcal{A})$ is the $i$-th Betti number of $V \backslash \cup_{H \in \mathcal{A}} H$ when $\mathbb{K}=\mathbb{C}$.

Let $\theta_{E}$ be the Euler derivation $\theta_{E}=\sum_{i=1}^{\ell} x_{i} \partial_{x_{i}}$ which is always contained in $D(\mathcal{A})$. Thus by $(1.2), \operatorname{deg} \theta_{E}=1$ is contained in $\exp (\mathcal{A})$ if $\mathcal{A} \neq \emptyset$ is free. The localization $\mathcal{A}_{X}$ is free if $\mathcal{A}$ is free for any $X \in L(\mathcal{A})$ (see [16], Theorem 4.37). We say that $\mathcal{A}$ is locally free if $\mathcal{A}_{X}$ is free for any $X \in L(\mathcal{A})$ with $X \neq \bigcap_{H \in \mathcal{A}} H$. $\mathcal{A}$ is locally free if and only if the sheaf $\widetilde{D_{H}(\mathcal{A})}$ is a vector bundle on $\mathbf{P}^{\ell-1}=\mathbf{P}\left(V^{*}\right)=\operatorname{Proj}(S)$ for any (equivalently, some) $H \in \mathcal{A}$ (see [23, Proposition 1.20).

Define the Euler restriction map $\rho: D(\mathcal{A}) \rightarrow D\left(\mathcal{A}^{H}\right)$ of an arrangement $\mathcal{A}$, by taking modulo $\alpha_{H}$. Then we have the following:

Proposition 2.1 (e.g., Proposition 4.45 in [16])

Let $H \in \mathcal{A}$. Then there is an exact sequence

$$
0 \rightarrow D(\mathcal{A} \backslash\{H\}) \stackrel{\alpha_{H}}{\rightarrow} D(\mathcal{A}) \stackrel{\rho}{\rightarrow} D\left(\mathcal{A}^{H}\right) .
$$

The following is the most important, and relates algebra, topology and combinatorics of free arrangements.

Theorem 2.2 (Terao's factorization, [19])

Assume that $\mathcal{A}$ is free with $\exp (\mathcal{A})=\left(d_{1}, \ldots, d_{\ell}\right)$. Then $\chi(\mathcal{A} ; t)=\prod_{i=1}^{\ell}(t-$ $\left.d_{i}\right)$.

Let us collect several fundamental results on freeness.

Proposition 2.3 ([18])

Let $H \in \mathcal{A}$. Then there is a polynomial $B$ of degree $|\mathcal{A}|-\left|\mathcal{A}^{H}\right|-1$ such that $\alpha_{H} \nmid B$, and

$$
\theta\left(\alpha_{H}\right) \in\left(\alpha_{H}, B\right)
$$

for all $\theta \in D(\mathcal{A} \backslash\{H\})$.

Theorem 2.4 ([18], Terao's addition-deletion theorem)

Let $H \in \mathcal{A}, \mathcal{A}^{\prime}:=\mathcal{A} \backslash\{H\}$ and let $\mathcal{A}^{\prime \prime}:=\mathcal{A}^{H}$. Then two of the following imply the third:

(1) $\mathcal{A}$ is free with $\exp (\mathcal{A})=\left(d_{1}, \ldots, d_{\ell-1}, d_{\ell}\right)$. 
(2) $\mathcal{A}^{\prime}$ is free with $\exp \left(\mathcal{A}^{\prime}\right)=\left(d_{1}, \ldots, d_{\ell-1}, d_{\ell}-1\right)$.

(3) $\mathcal{A}^{\prime \prime}$ is free with $\exp \left(\mathcal{A}^{\prime \prime}\right)=\left(d_{1}, \ldots, d_{\ell-1}\right)$.

Moreover, all the three hold true if $\mathcal{A}$ and $\mathcal{A}^{\prime}$ are both free.

Theorem 2.5 ([2], Division theorem)

Let $H \in \mathcal{A}$. Then $\mathcal{A}$ is free if $\mathcal{A}^{H}$ is free and $\pi\left(\mathcal{A}^{H} ; t\right) \mid \pi(\mathcal{A} ; t)$.

The following is the special case of Theorem 2.5 when $\ell=3$.

Proposition 2.6 ([1], Theorem 1.1)

Let $\ell=3$ and $\chi(\mathcal{A} ; t)=(t-1)(t-a)(t-b)$ with two real numbers $a \leq b$. For a plane $L$ which may or may not be in $\mathcal{A}$, let $n_{L}:=\mid\{H \cap L \neq \emptyset \mid H \in$ $\mathcal{A}, H \neq L\} \mid$. Then $n_{L} \leq a+1$ or $b+1 \leq n_{L}$. Moreover, $\mathcal{A}$ and $\mathcal{A}^{\prime}$ are both free if the equality holds.

Next let us recall the theory of multiarrangements. A pair $(\mathcal{A}, m)$ is a multiarrangement if $m: \mathcal{A} \rightarrow \mathbb{Z}_{\geq 1}$. Let $|m|:=\sum_{H \in \mathcal{A}} m(H)$ and $Q(\mathcal{A}, m):=\prod_{H \in \mathcal{A}} \alpha_{H}^{m(H)}$. For two multiplicities $m$ and $k$ on $\mathcal{A}, k \leq m$ stands for $k(H) \leq m(H)$ for all $H \in \mathcal{A}$. For $H \in \mathcal{A}$, let $\delta_{H}: \mathcal{A} \rightarrow\{0,1\}$ be the characteristic multiplicity defined by $\delta_{H}(L)=1$ if $L=H$, and 0 otherwise. We may define its logarithmic derivation module $D(\mathcal{A}, m)$ as

$$
D(\mathcal{A}, m):=\left\{\theta \in \operatorname{Der} S \mid \theta\left(\alpha_{H}\right) \in S \alpha_{H}^{m(H)}(\forall H \in \mathcal{A})\right\} .
$$

$D(\mathcal{A}, m)$ is a reflexive graded $S$-module, but not free in general. Thus we may define the freeness and exponents of $(\mathcal{A}, m)$ in the same way as for $m \equiv 1$. By the reflexivity, $(\mathcal{A}, m)$ is free when $\ell=2$. We can also define the localization $\left(\mathcal{A}_{X}, m_{X}\right)$ of the multiarrangement $(\mathcal{A}, m)$ at $X \in L(\mathcal{A})$ by $m_{X}:=\left.m\right|_{\mathcal{A}_{X}}$. Then $\left(\mathcal{A}_{X}, m_{X}\right)$ is free if $(\mathcal{A}, m)$ is free as when $m \equiv 1$. The most fundamental criterion for freeness is the following.

Theorem 2.7 (Saito's criterion, [17], 24])

Let $\theta_{1}, \ldots, \theta_{\ell}$ be homogeneous elements in $D(\mathcal{A}, m)$. Then $D(\mathcal{A}, m)$ has a free basis $\theta_{1}, \ldots, \theta_{\ell}$ if and only if they are $S$-independent, and $|m|=\sum_{i=1}^{\ell} \operatorname{deg} \theta_{i}$. Also, for the matrix $M:=\left(\theta_{i}\left(x_{j}\right)\right)$, the above two statements are equivalent to

$$
\operatorname{det} M=c Q(\mathcal{A}, m)
$$

for some $c \in \mathbb{K} \backslash\{0\}$.

We can construct the multiarrangement canonically from an arrangement $\mathcal{A}$ in the following manner: 
Definition $2.8([24])$

For an arrangement $\mathcal{A}$ in $\mathbb{K}^{\ell}$ and $H \in \mathcal{A}$, define the Ziegler multiplicity $m^{H}: \mathcal{A}^{H} \rightarrow \mathbb{Z}_{>0}$ by $m^{H}(X):=|\{L \in \mathcal{A} \backslash\{H\} \mid L \cap H=X\}|$ for $X \in \mathcal{A}^{H}$. The pair $\left(\mathcal{A}^{H}, m^{H}\right)$ is called the Ziegler restriction of $\mathcal{A}$ onto $H$. Also, there is a Ziegler restriction map

$$
\pi: D_{H}(\mathcal{A}) \rightarrow D\left(\mathcal{A}^{H}, m^{H}\right)
$$

by taking modulo $\alpha_{H}$. In particular, there is an exact sequence

$$
0 \rightarrow D_{H}(\mathcal{A}) \stackrel{\cdot \alpha_{H}}{\rightarrow} D_{H}(\mathcal{A}) \stackrel{\pi}{\rightarrow} D\left(\mathcal{A}^{H}, m^{H}\right)
$$

By definition, $\left|m^{H}\right|=|\mathcal{A}|-1$. A remarkable property of Ziegler restriction maps is the following.

\section{Theorem $2.9([24])$}

Assume that $\mathcal{A}$ is free with $\exp (\mathcal{A})=\left(1, d_{2}, \ldots, d_{\ell}\right)$. Then for any $H \in \mathcal{A}$, the Ziegler restriction $\left(\mathcal{A}^{H}, m^{H}\right)$ is also free with $\exp \left(\mathcal{A}^{H}, m^{H}\right)=\left(d_{2}, \ldots, d_{\ell}\right)$. Explicitly, for the Ziegler restriction $\pi: D_{H}(\mathcal{A}) \rightarrow D\left(\mathcal{A}^{H}, m^{H}\right)$, there is a basis $\theta_{2}, \ldots, \theta_{\ell}$ for $D_{H}(\mathcal{A})$ such that $\pi\left(\theta_{2}\right), \ldots, \pi\left(\theta_{\ell}\right)$ form a basis for $D\left(\mathcal{A}^{H}, m^{H}\right)$ (recall the decomposition (1.2)). In particular, $\pi$ is surjective when $\mathcal{A}$ is free.

The converse of Theorem 2.9 is proved by Yoshinaga. In this article we mainly use it when $\ell=3$.

Theorem 2.10 (Yoshinaga's criterion, [22])

Let $\ell=3$ and $H \in \mathcal{A}$. Let $\chi_{0}(\mathcal{A} ; t)=t^{2}-(|\mathcal{A}|-1) t+b_{2}^{0}(\mathcal{A})$, and $\exp \left(\mathcal{A}^{H}, m^{H}\right)=\left(d_{1}, d_{2}\right)$. Then $b_{2}^{0}(\mathcal{A}) \geq d_{1} d_{2}$, and $\mathcal{A}$ is free if and only if $b_{2}^{0}(\mathcal{A})=d_{1} d_{2}$. Moreover, $b_{2}^{0}(\mathcal{A})-d_{1} d_{2}=\operatorname{dim}_{\mathbb{K}} \operatorname{coker}\left(\pi: D_{H}(\mathcal{A}) \rightarrow\right.$ $\left.D\left(\mathcal{A}^{H}, m^{H}\right)\right)<+\infty$.

Also, the following relation between Betti numbers and Chern classe are important.

Proposition 2.11 ([11], Proposition 5.18)

$b_{i}^{0}(\mathcal{A})=(-1)^{i} c_{i}\left(\widehat{\left.D_{0}(\mathcal{A})\right)}\right.$ for $i=0,1,2$.

Now we introduce the following variant of the addition theorem.

Theorem 2.12 (Multiple addition theorem (MAT), [5])

Let $\mathcal{A} \ni H, \mathcal{A}^{\prime}:=\mathcal{A} \backslash\{H\}$. Assume that $\mathcal{A}^{\prime}$ is free with $\exp \left(\mathcal{A}^{\prime}\right)=$ $\left(d_{1}, \ldots, d_{\ell}\right)_{\leq}$. If $d_{\ell}=\left|\mathcal{A}^{\prime}\right|-\left|\mathcal{A}^{H}\right|$, then $\mathcal{A}$ is free with $\exp (\mathcal{A})=\left(d_{1}, \ldots, d_{\ell-1}, d_{\ell}+\right.$ $1)$. 
The following is due to Hoge (unpublished, [15]), which is close to but different from MAT. We give a proof due to Hoge for the completeness.

Theorem $2.13([15])$

Let $\mathcal{A} \ni H, \mathcal{A}^{\prime}:=\mathcal{A} \backslash\{H\}$. Assume that $\mathcal{A}^{\prime}$ is free with $\exp \left(\mathcal{A}^{\prime}\right)=$ $\left(d_{1}, \ldots, d_{\ell}\right)_{<}$. If $d_{\ell} \geq\left|\mathcal{A}^{\prime}\right|-\left|\mathcal{A}^{H}\right|=: d \geq d_{\ell-1}$, then $\mathcal{A}$ is free with $\exp (\mathcal{A})=$ $\left(d_{1}, \ldots, d_{\ell-2}, d_{\ell-1}+1, d_{\ell}\right)$. In fact, $d_{\ell-1}<d<d_{\ell}$ cannot occur.

Proof. When $d=d_{\ell}$, this is nothing but Theorem 2.12. Assume that $d_{\ell-1} \leq$ $d<d_{\ell}$. Let $\theta_{1}, \ldots, \theta_{\ell}$ be a homogeneous basis for $D\left(\mathcal{A}^{\prime}\right)$ with $\operatorname{deg} \theta_{i}=d_{i}$. By Proposition 2.3, there exists a polynomial $B \in S_{d}$ such that $\theta\left(\alpha_{H}\right) \in\left(\alpha_{H}, B\right)$ for $\theta \in D\left(\mathcal{A}^{\prime}\right)$. Let $\theta_{i}\left(\alpha_{H}\right)=a_{i} \alpha_{H}+b_{i} B$ for $i=1, \ldots, \ell$ by Proposition 2.3. Assume that there is some $i<\ell$ such that $\theta_{i} \notin D(\mathcal{A})$. We may assume that $i=\ell-1$. This is the same as $b_{\ell-1}=c \in \mathbb{K} \backslash\{0\}$. By replacing $\theta_{\ell-1}$ by $\theta_{\ell-1} / c$, we may assume that $c=1$. Thus replacing $\theta_{i}$ by $\theta_{i}-b_{i} \theta_{\ell-1}$, it holds that $\theta_{i} \in D(\mathcal{A})$ unless $i=\ell-1$. Hence Theorem 2.7 implies that $\theta_{1}, \ldots, \theta_{\ell-2}, \alpha_{H} \theta_{\ell-1}, \theta_{\ell}$ form a basis for $D(\mathcal{A})$. By Theorem 2.4, this implies that $d=d_{\ell-1}$.

Hence we may assume that $\theta_{i} \in D(\mathcal{A})$ if $i<\ell$. Then again Theorem 2.7 implies that $\theta_{1}, \ldots, \theta_{\ell-1}, \alpha_{H} \theta_{\ell}$ form a basis for $D(\mathcal{A})$, and $d=d_{\ell}$ by Theorem 2.4, which is a contradiction.

\section{Remark 2.14}

For $H \in \mathcal{A}$ and $S^{\prime}:=S / \alpha_{H} S$, let $\bar{f}$ denote the image of $f \in S$ by the canonical surjection $S \rightarrow S^{\prime}$. By abuse of notations, $\bar{f}$ is also denoted by $\rho(f)$ or $\pi(f)$ respectively, where $\rho$ and $\pi$ are the Euler and Ziegler restrictions of $\mathcal{A}$ with respect to $H$.

\section{Replacement and locally free tricks}

In this section we introduce two important techniques used for the rest of this article frequently. The first key is the following easy lemma.

\section{Lemma 3.1}

Let $H \in \mathcal{A}$ and $d:=|\mathcal{A}|-\left|\mathcal{A}^{H}\right|$. Let $\pi: D_{H}(\mathcal{A}) \rightarrow D\left(\mathcal{A}^{H}, m^{H}\right)$ be the Ziegler restriction, and $\rho: D(\mathcal{A}) \rightarrow D\left(\mathcal{A}^{H}\right)$ the Euler restriction. Then

(1) $\left.\rho\right|_{D_{H}(\mathcal{A})}=\pi$.

(2) Let $t: \mathcal{A}^{H} \rightarrow \mathcal{A} \backslash\{H\}$ be a section such that $t(X) \cap H=X$ for all $X \in \mathcal{A}^{H}$. Define $Q^{\prime}:=\prod_{X \in \mathcal{A}^{H}} \alpha_{t(X)}^{m^{H}(X)-1} \in S_{d-1}$ and $\theta_{E}^{H}:=\rho\left(Q^{\prime} \theta_{E}\right)$. Then $\theta_{E}^{H} \in D\left(\mathcal{A}^{H}, m^{H}\right)_{d}$. 
Proof. Immediate by definitions.

The following has been well-known and used by specialists. Here we put it since we also use this frequently.

\section{Proposition 3.2}

Let $H \in \mathcal{A}, \theta \in D(\mathcal{A} \backslash\{H\})$ with $\operatorname{deg} \theta=d:=\left|\mathcal{A}^{\prime}\right|-\left|\mathcal{A}^{H}\right|$. Assume that $\theta \notin D(\mathcal{A})$. Then for all $\varphi \in D\left(\mathcal{A}^{\prime}\right)$, there is $f \in S$ such that $\varphi-f \theta \in D(\mathcal{A})$.

Proof. Let $B \in S_{d}$ be the polynomial in Proposition 2.3. Thus we may put $\theta\left(\alpha_{H}\right)=g \alpha_{H}+B, \varphi\left(\alpha_{H}\right)=h \alpha_{H}+f B$ with $f, g, h \in S$. Then the statement is clear.

The following is a corollary of Proposition 3.2 .

\section{Corollary 3.3}

Let $H \in \mathcal{A}$. Assume that there exists $\varphi \in D(\mathcal{A} \backslash\{H\})_{|\mathcal{A}|-\left|\mathcal{A}^{H}\right|-1}$ such that $\varphi \notin D(\mathcal{A})$. Then

$$
D\left(\mathcal{A}^{\prime}\right)=D(\mathcal{A})+S \varphi
$$

The first technique on freeness is the following replacement trick, which was first shown in [7], Propositon 2.7.

\section{Theorem 3.4 (Proposition 2.7, [7])}

Let $\mathcal{A}$ be free with $\exp (\mathcal{A})=\left(1, d_{2}, \ldots, d_{\ell}\right)_{\leq}, H \in \mathcal{A}$, and let $\theta_{E}, \theta_{2}, \ldots, \theta_{\ell}$ be a basis for $D(\mathcal{A})$ with $\theta_{i} \in D_{H}(\mathcal{A})$ and $\operatorname{deg} \theta_{i}=d_{i}$. Assume that $\theta_{E}^{H}$ can be expressed as

$$
\theta_{E}^{H}=\sum_{i=2}^{\ell} \pi\left(f_{i} \theta_{i}\right),
$$

and $\pi\left(f_{k}\right) \neq 0$ for some $k$ with $d_{k}=d:=|\mathcal{A}|-\left|\mathcal{A}^{H}\right|$. Then $\mathcal{A}^{\prime}:=\mathcal{A} \backslash\{H\}$ and $\mathcal{A}^{H}$ are both free.

The next one works when we consider $D(\mathcal{A})$ generated by $(\ell+1)$-elements and $\mathcal{A}$ is locally free. We call this the locally free trick, which was first used in [4] implicitly.

\section{Theorem 3.5}

Assume that $\mathcal{A}$ is locally free but not free, and $D(\mathcal{A})$ has a minimal free resolution of the following form:

$$
0 \rightarrow S[-d-1] \stackrel{F}{\rightarrow} \oplus_{i=1}^{\ell+1} S\left[-d_{i}\right] \stackrel{G}{\rightarrow} D(\mathcal{A}) \rightarrow 0 .
$$


Let $F=\left(f_{1}, \ldots, f_{\ell}, f_{\ell+1}\right)$ and $G=\left(\theta_{E}, \theta_{2}, \ldots, \theta_{\ell}, \theta_{\ell+1}\right)$. Then $f_{i} \neq 0$ for all $i=2, \ldots, \ell+1$.

Proof. Assume that $f_{\ell+1}=0$. Then the relation among $\theta_{E}, \theta_{2}, \ldots, \theta_{\ell+1}$ is of the form

$$
f_{1} \theta_{E}+\sum_{i=2}^{\ell} f_{i} \theta_{i}=0 .
$$

Since the resolution is minimal, we may assume that $\operatorname{deg} f_{i}>0$ for all $i$ if $f_{i} \neq 0$. Recall that the freeness is independent of the extension of the base field $\mathbb{K}$ (see [16], the first paragraph in page 151). So we may assume that $\mathbb{K}$ is an infinite field. Now consider the zero locus

$$
Z:=\cap_{i=2}^{\ell}\left\{f_{i}=0\right\} \subset \mathbf{P}(V)=\mathbf{P}^{\ell-1} .
$$

This is not empty since it is the intersection of at most $(\ell-1)$-hypersurfaces in $\mathbf{P}^{\ell-1}$. Since $\theta_{E}$ is a nowhere vanishing vector field, it holds that $Z=$ $Z \cap\left\{f_{1}=0\right\}$. Take a point $x \in Z$ and let $k_{x}$ be the residue field of $\mathcal{O}_{\mathbf{P}^{\ell-1}}$ at $x$. Note that $D(\mathcal{A}) \otimes k_{x}=k_{x}^{\ell}$ since $\mathcal{A}$ is locally free. Thus tensoring $k_{x}$ to the minimal free resolution, we obtain the exact sequence

$$
k_{x} \stackrel{\left.F\right|_{x}=0}{\rightarrow} k_{x}^{\ell+1} \rightarrow k_{x}^{\ell} \rightarrow 0
$$

a contradiction.

\section{Proofs of main results}

In this section we prove the main results posed in $\S 1$. First let us prove fundamental properties of plus-one generated arrangements.

\section{Proposition 4.1}

Let $\mathcal{A}$ be plus-one generated with $\operatorname{PO} \exp (\mathcal{A})=\left(d_{1}, \ldots, d_{\ell}\right)$ and level $d$. Then $b_{1}(\mathcal{A})=|\mathcal{A}|=\sum_{i=2}^{\ell} d_{i}$, and $b_{2}(\mathcal{A})=\sum_{2 \leq i<j \leq \ell} d_{i} d_{j}+d$.

Proof. Immediate from Definition 1.1 and Proposition 2.11.

\section{Proposition 4.2}

Let $\mathcal{A}$ be plus-one generated with the minimal set of homogeneous generators $\theta_{1}=\theta_{E}, \theta_{2}, \ldots, \theta_{\ell}, \varphi=: \theta_{\ell+1}$ with the relation

$$
\sum_{i=1}^{\ell+1} f_{i} \theta_{i}=0 .
$$


Let $I:=\left\{i \mid 1 \leq i \leq \ell+1, f_{i} \neq 0\right\}$. Then for any $j \in I$,

$$
\left\{\theta_{i}\right\}_{i \in I \backslash\{j\}} \cup\left\{\theta_{k}\right\}_{k \notin I}
$$

are $S$-independent.

Proof. Since there is the unique relation among $D(\mathcal{A})$, and $\operatorname{rank}_{S} D(\mathcal{A})=\ell$, it is obvious.

Proof of Theorem 1.4. Let $\theta_{E}=\theta_{1}, \theta_{2}, \ldots, \theta_{\ell}$ be a basis for $D(\mathcal{A})$ such that $\operatorname{deg} \theta_{i}=d_{i}, d_{1}=1 \leq d_{2} \leq \cdots \leq d_{\ell}$ and $\theta_{i} \in D_{H}(\mathcal{A})(i \geq 2)$. Let $\pi$ : $D_{H}(\mathcal{A}) \rightarrow D\left(\mathcal{A}^{H}, m^{H}\right)$ be the Ziegler restriction map, thus $\pi\left(\theta_{2}\right), \ldots, \pi\left(\theta_{\ell}\right)$ form a basis for $D\left(\mathcal{A}^{H}, m^{H}\right)$ by Theorem 2.8. Let $Q^{\prime}:=\prod_{X \in \mathcal{A}^{H}} \alpha_{t(X)}^{m^{H}(X)-1} \in$ $S$, where $t: \mathcal{A}^{H} \rightarrow \mathcal{A} \backslash\{H\}$ is a section as in Lemma 3.1. Then Lemma 3.1 implies that $\rho\left(Q^{\prime} \theta_{E}\right) \in D\left(\mathcal{A}^{H}, m^{H}\right)_{d}$, where $d:=|\mathcal{A}|-\left|\mathcal{A}^{H}\right|$ and $\rho: D(\mathcal{A}) \rightarrow$ $D\left(\mathcal{A}^{H}\right)$ is the Euler restriction map. Hence there are polynomials $f_{i} \in S$ such that

$$
\rho\left(Q^{\prime} \theta_{E}\right)=\sum_{i=2}^{\ell} \pi\left(f_{i} \theta_{i}\right)
$$

Assume that $\mathcal{A}^{\prime}$ is not free. By Theorem [3.4, we may assume that $d_{i}=0$ if $d_{i}=d$. Hence in the expression

$$
\rho\left(Q^{\prime} \theta_{E}\right)=\sum_{i=2}^{\ell} \pi\left(f_{i} \theta_{i}\right)=\sum_{i=2}^{\ell} \rho\left(f_{i} \theta_{i}\right)
$$

we have that $\pi\left(f_{i}\right)=0$ if $d_{i} \geq d$. Here we used Lemma 3.1. Let $k$ be the minimal integer such that $d_{k+1} \geq d$. By Proposition 2.1, there is $\varphi \in$ $D\left(\mathcal{A}^{\prime}\right)_{d-1}$ such that

$$
\alpha_{H} \varphi=\sum_{i=2}^{k} f_{i} \theta_{i}-Q^{\prime} \theta_{E}
$$

We prove that

$$
D\left(\mathcal{A}^{\prime}\right)=\left\langle\theta_{E}, \theta_{2}, \ldots, \theta_{\ell}, \varphi\right\rangle_{S}
$$

Note that

$$
\sum_{i=2}^{k} f_{i} \theta_{i}\left(\alpha_{H}\right)-Q^{\prime} \theta_{E}\left(\alpha_{H}\right)=-\alpha_{H} Q^{\prime}=\alpha_{H} \varphi\left(\alpha_{H}\right) \neq 0
$$

and $\alpha_{H} \nmid Q^{\prime}$. Hence $\varphi \neq 0$ and $\varphi\left(\alpha_{H}\right) \notin S \alpha_{H}$, i.e., $\varphi \notin D(\mathcal{A})$. By Proposition 3.2. for all $\theta \in D\left(\mathcal{A}^{\prime}\right)$, there is $f \in S$ such that $\theta-f \varphi \in D(\mathcal{A})$. Hence $\theta \in$ 
$\left\langle\theta_{E}, \theta_{2}, \ldots, \theta_{\ell}, \varphi\right\rangle_{S}$. Now let us determine relations among these generators. Recall that we have one relation:

$$
\alpha_{H} \varphi=\sum_{i=2}^{k} f_{i} \theta_{i}-Q^{\prime} \theta_{E} .
$$

Let

$$
g^{\prime} \varphi=\sum_{i=2}^{\ell} g_{i} \theta_{i}-h \theta_{E}
$$

be another relation. Since the right hand side is in $D(\mathcal{A})$ and $\varphi \notin D(\mathcal{A})$, we may assume that $g^{\prime}=\alpha_{H} g$. Then

$$
\begin{aligned}
g\left(\alpha_{H} \varphi\right) & =g\left(\sum_{i=2}^{k} f_{i} \theta_{i}-Q^{\prime} \theta_{E}\right) \\
& =\sum_{i=2}^{\ell} g_{i} \theta_{i}-h \theta_{E} .
\end{aligned}
$$

Since $\theta_{E}, \theta_{2}, \ldots, \theta_{\ell}$ form a basis for $D(\mathcal{A})$, it holds that it holds that $g f_{i}=g_{i}$ and $g Q^{\prime}=h$. Hence the relation is unique.

Proof of Theorem 1.7. Almost all parts follow from Theorems 1.4, 2.4 and 2.5. To complete the proof, it suffices to show that the condition (2) implies the freeness of $\mathcal{A}$. Let $\theta_{1}=\theta_{E}, \theta_{2}, \ldots \theta_{\ell}, \varphi$ be a minimal set of homogeneous generators for $D\left(\mathcal{A}^{\prime}\right)$ such that $\operatorname{deg} \theta_{i}=d_{i}, \operatorname{deg} \varphi=d$ and

$$
\sum_{i=1}^{\ell} f_{i} \theta_{i}+\alpha \varphi=0 \text {. }
$$

By the assumption, $\alpha \neq 0, \theta_{i} \in D(\mathcal{A})$ and $\varphi \notin D(\mathcal{A})$. Thus $\alpha=\alpha_{H}$. Since $\theta_{1}, \ldots, \theta_{\ell}$ are $S$-independent by Proposition 4.2 , and $\sum_{i=1}^{\ell} d_{i}=|\mathcal{A}|$ by Proposition 4.1, Theorem 2.7 completes the proof.

Proof of Theorem 1.8. Note that $\mathcal{A}^{\prime}$ is not free by Theorem 2.4. The "only if" part follows from Theorems 1.4 and 2.5. It suffices to show the "if" part. Let $\theta_{1}=\theta_{E}, \theta_{2}, \ldots, \theta_{\ell}$ and $\varphi$ be a generator for $D\left(\mathcal{A}^{\prime}\right)$ such that $\operatorname{deg} \theta_{i}=d_{i}<d$. Note that $\varphi$ is a level element, but $\operatorname{deg} \varphi$ is not clear. However, since $d=\left|\mathcal{A}^{\prime}\right|-\left|\mathcal{A}^{H}\right|>d_{i}$ for all $i$, Proposition 2.3 implies that $\theta_{i} \in D(\mathcal{A})$ for all $i$. Thus $D\left(\mathcal{A}^{\prime}\right) \supsetneq D(\mathcal{A})$ shows that $\operatorname{deg} \varphi \geq d>d_{i}$. By the assumption,

$$
\sum_{i=1}^{\ell} f_{i} \theta_{i}+\alpha \varphi=0 \quad\left(0 \neq \alpha \in V^{*}\right) .
$$


Since $\theta_{1}, \ldots, \theta_{\ell}$ are $S$-independent by Proposition 4.2, and $\sum_{i=1}^{\ell} d_{i}=|\mathcal{A}|$ by Proposition 4.1, Theorem 2.7 completes the proof.

Proof of Theorem 1.9. Let $\alpha_{H}=: x_{1}$ and $\theta_{1}=\theta_{E}, \theta_{2}, \ldots, \theta_{\ell}$ form a basis for $D\left(\mathcal{A}^{\prime}\right)$ with $\operatorname{deg} \theta_{i}=d_{i}$. Assume that $\theta_{\ell-2} \notin D(\mathcal{A})$. Then Proposition 2.3 implies that $d=\left|\mathcal{A}^{\prime}\right|-\left|\mathcal{A}^{H}\right|=d_{\ell-2}$, and we may assume that $\theta_{\ell-1}, \theta_{\ell} \in D(\mathcal{A})$ by Proposition 3.2. Hence $\mathcal{A}$ is free with basis $\theta_{E}, \theta_{2}, \ldots, \theta_{\ell-3}, \alpha_{H} \theta_{\ell-2}, \theta_{\ell-1}, \theta_{\ell}$. So we may assume that $\theta_{\ell-2} \in D(\mathcal{A})$. If $\theta_{\ell-1} \notin D(\mathcal{A})$ and $\theta_{\ell} \in D(\mathcal{A})$, then Theorem 2.13 confirms that $\mathcal{A}$ is free. The same holds if $\theta_{\ell} \notin D(\mathcal{A})$ and $\theta_{\ell-1} \in D(\mathcal{A})$ by Theorem 2.12 .

As a conclusion, the rest case is when $\theta_{j} \in D(\mathcal{A})(\forall j \leq \ell-2)$ and $\theta_{i} \notin D(\mathcal{A})(i=\ell-1, \ell)$ by Proposition 2.3. So again by Proposition 2.3, we may assume that $d_{\ell-2} \leq d<d_{\ell-1}$. Let $B$ be a polynomial of degree $d$ satisfying

$$
D\left(\mathcal{A}^{\prime}\right)\left(x_{1}\right) \subset\left(x_{1}, B\right)
$$

as in Proposition 2.3. We may assume that $B \in \mathbb{K}\left[x_{2}, \ldots, x_{\ell}\right]$. From now on, $\equiv$ implies to take modulo $x_{1}$. Let $D_{1}:=\oplus_{i=1}^{\ell-2} S \theta_{i} \subset D(\mathcal{A})$. Then we have a canonical surjection $D(\mathcal{A}) \rightarrow D_{1}$ as a composition of

$$
D(\mathcal{A}) \subset D\left(\mathcal{A}^{\prime}\right) \rightarrow D_{1} \rightarrow 0
$$

since $D\left(\mathcal{A}^{\prime}\right)$ contains $D_{1}$ as a direct summand. Since this map has a canonical section too, it holds that

$$
D(\mathcal{A})=D_{1} \oplus D_{2} \subset D\left(\mathcal{A}^{\prime}\right)=D_{1} \oplus D_{2}^{\prime},
$$

where $D_{2}^{\prime}=S \theta_{\ell-1} \oplus S \theta_{\ell} \supset D_{2}$. Let $\theta_{i}\left(x_{1}\right) \equiv f_{i} B(i=\ell-1, \ell)$. Since $\theta_{i}\left(x_{1}\right) \in\left(x_{1}, B\right)$, we may again assume that $f_{i} \in \mathbb{K}\left[x_{2}, \ldots, x_{\ell}\right]=: S^{\prime} \subset S$. Let $\left(f_{\ell-1}, f_{\ell}\right) \equiv g \in S^{\prime}$ and $f_{i}=h_{i} g\left(h_{i} \in S^{\prime}\right)$. By the choice of $f_{i},\left(f_{\ell-1}, f_{\ell}\right)=$ $g \in S$ too. Now let us show that

$$
D_{2}^{\prime \prime}:=\left(x_{1} \theta_{\ell-1}, x_{1} \theta_{\ell}, h_{\ell} \theta_{\ell-1}-h_{\ell-1} \theta_{\ell}\right)_{S}=D_{2} .
$$

By definition it is clear that $D_{2}^{\prime \prime} \subset D_{2}$. It suffices to show that $D_{2}^{\prime \prime} \supset D_{2}$. Let $D_{2} \ni \theta=s \theta_{\ell-1}+t \theta_{\ell}$. If $x_{1} \mid s$ and $x_{1} \mid t$, then there is nothing to show. Assume that $x_{1} \nmid s$. Since $\theta\left(x_{1}\right) \in S x_{1}$ and $\theta_{i}\left(x_{1}\right) \notin S x_{1}(i=\ell-1, \ell)$, we have $x_{1} \nmid t$. Take a modulo by $x_{1}$ of $\theta\left(x_{1}\right)=s \theta_{\ell-1}\left(x_{1}\right)+t \theta_{\ell}\left(x_{1}\right)$ to obtain that

$$
0 \equiv s f_{\ell-1} B+t f_{\ell} B
$$

in $S / x_{1} S$. Since $B \neq 0 \neq g$ in $S / x_{1} S$, we have

$$
s h_{\ell-1} \equiv-t h_{\ell}
$$


implying that $s \equiv h_{\ell} u, t \equiv-h_{\ell-1} u$ for some $u \in S$, which shows that $\mathcal{A}$ is strictly plus-one generated with $\operatorname{PO} \exp (\mathcal{A})=\left(d_{1}, \ldots, d_{\ell-2}, d_{\ell-1}+1, d_{\ell}+1\right)$.

Finally let us determine the level $c=\operatorname{deg} h_{\ell} \theta_{\ell-1}$. Let $\operatorname{deg} g=: a$. Then $\operatorname{deg} h_{\ell}=d_{\ell}-a-d$, where $\operatorname{deg} B=d$. Thus $c=d_{\ell-1}+d_{\ell}-a-d$. Now compute $b_{2}(\mathcal{A})$ in two ways. First, by the deletion-restriction formula,

$$
b_{2}(\mathcal{A})=b_{2}\left(\mathcal{A}^{\prime}\right)+\left|\mathcal{A}^{H}\right| \text {. }
$$

Thus

$$
b_{2}(\mathcal{A})=\sum_{2 \leq i<j \leq \ell} d_{i} d_{j}+2 \sum_{i=2}^{\ell} d_{i}+1-d .
$$

On the other hand, by Proposition 4.1,

$$
\begin{aligned}
b_{2}(\mathcal{A}) & =\sum_{2 \leq i<j \leq \ell} d_{i} d_{j}+2 \sum_{i=2}^{\ell-2} d_{i}+d_{\ell-1}+d_{\ell}+1+c \\
& =\sum_{2 \leq i<j \leq \ell} d_{i} d_{j}+2 \sum_{i=2}^{\ell} d_{i}+1-a-d . \\
0 \Longleftrightarrow & =1 \Longleftrightarrow\left(f_{\ell-1}, f_{\ell}\right)=1, \text { and } \\
c= & d_{\ell-1}+d_{\ell}-d=d_{\ell-1}+d_{\ell}-|\mathcal{A}|+\left|\mathcal{A}^{H}\right|+1
\end{aligned}
$$

Hence $a=0 \Longleftrightarrow g=1 \Longleftrightarrow\left(f_{\ell-1}, f_{\ell}\right)=1$, and

as desired.

An easy conclusion is the following.

\section{Corollary 4.3}

Assume that $\mathcal{A}$ is free with $\exp (\mathcal{A})=\left(d_{1}, d_{2}, \ldots, d_{\ell}\right)_{\leq}$with $d_{1}=1$, and $H \in \mathcal{A}$. If $\mathcal{A}^{\prime}:=\mathcal{A} \backslash\{H\}$ is locally free, then $d:=|\mathcal{A}|-\left|\mathcal{A}^{H}\right|=d_{i}$ for some $i$, or $d>d_{\ell}$.

Proof. Since $\theta_{E}^{H} \in D\left(\mathcal{A}^{H}, m^{H}\right)_{d}$, it holds that $d \geq d_{2}$. Assume that $d_{k}<$ $d<d_{k+1}$ for some $2 \leq k \leq \ell-1$. By Theorem 1.4, $D\left(\mathcal{A}^{\prime}\right)$ is generated by $\theta_{E}, \theta_{2}, \ldots, \theta_{\ell}$, a basis for $D(\mathcal{A})$, and $\varphi \in D\left(\mathcal{A}^{\prime}\right)_{d-1}$. There are the unique relation

$$
\alpha_{H} \varphi=Q^{\prime} \theta_{E}+\sum_{i=2}^{\ell} f_{i} \theta_{i} .
$$

By the assumption on $d=\operatorname{deg} \alpha_{H} \varphi$, at least $f_{\ell}=0$, which contradicts Theorem 3.5.

As far as we investigated, the conclusion in Corollary 4.3 holds true without the assumption of local freeness of $\mathcal{A}^{\prime}$ (see $\S 7$ for example). So let us pose the following conjecture. 


\section{Conjecture 4.4}

Let $\mathcal{A}$ be free with $\exp (\mathcal{A})=\left(d_{1}, d_{2}, \ldots, d_{\ell}\right)_{\leq}$with $d_{1}=1$, and $H \in \mathcal{A}$. Then $d:=|\mathcal{A}|-\left|\mathcal{A}^{H}\right|=d_{i}$ for some $i$, or $d>d_{\ell}$.

By Proposition 2.6. Conjecture 4.4 is true if $\ell=3$. We can relate the number of small roots of the characteristic polynomials with a level of a plus-one generated arrangement in terms of the local freeness at some codimension.

\section{Theorem 4.5}

Let $\mathcal{A}$ be free, $H \in \mathcal{A}, \mathcal{A}^{\prime}:=\mathcal{A} \backslash\{H\}$ and let $d:=|\mathcal{A}|-\left|\mathcal{A}^{H}\right|$. Assume that $\mathcal{A}^{\prime}$ is locally free at codimension $k$ along $H$, i.e., $\mathcal{A}_{X}$ is free for all $X \in L_{k}(\mathcal{A})$ with $H \supset X$. Then $\chi(\mathcal{A} ; t)$ has $d$ as its root, or it has at least $(k+1)$-roots less than $d$.

Proof. Assume that $\chi(\mathcal{A} ; d) \neq 0$. Let $d_{1}=1 \leq d_{2} \leq \cdots \leq d_{\ell}$ be roots of $\chi(\mathcal{A} ; t)$ with $d_{s}<d<d_{s+1}$. Assume that $s<k+1$. By Theorem 1.4, there are a minimal set of homogeneous generators $\theta_{1}=\theta_{E}, \theta_{2}, \ldots, \theta_{\ell}, \varphi$ for $D\left(\mathcal{A}^{\prime}\right)$ such that $\operatorname{deg} \theta_{i}=d_{i}, \operatorname{deg} \varphi=d-1$ and

$$
f_{1} \theta_{E}=\sum_{i=2}^{s} f_{i} \theta_{i}+\alpha_{H} \varphi
$$

at degree $d$ for $f_{i} \in S$ by the reason of degrees. Then by the same proof of Theorem [3.5, the zero locus $\alpha_{H}=f_{2}=\cdots=f_{s}=0$ is not empty in $\mathbf{P}(V) \simeq \mathbf{P}^{\ell-1}$, and it coincides with $f_{1}=\alpha_{H}=f_{2}=\cdots=f_{s}=0$. Take a prime ideal $P$ from that locus. By definition, codim $P \leq s \leq k$. Let

$$
0 \rightarrow S[-d-1] \rightarrow \oplus_{i=1}^{\ell} S\left[-d_{i}\right] \oplus S[-d] \rightarrow D\left(\mathcal{A}^{\prime}\right) \rightarrow 0
$$

be the minimal free resolution of the plus-one generated arrangement $\mathcal{A}^{\prime}$. Tensoring the residue field $k_{P}$ at $P$ gives us the exact sequence

$$
k_{P} \stackrel{0}{\rightarrow} k_{P}^{\ell+1} \rightarrow k_{P}^{\ell} \rightarrow 0
$$

a contradiction.

Based on these main results and Corollary 1.6, it seems natural to pose the following conjectures and problems.

\section{Question 4.6}

Let $\mathcal{A} \ni H$. Then

(1) are there any relation between $\operatorname{pd}_{S} D(\mathcal{A})$ and $\operatorname{pd}_{S} D\left(\mathcal{A}^{\prime}\right)$ ?

(2) Are there any relation between the cardinalities of minimal sets of homogeneous generators for $D(\mathcal{A})$ and $D\left(\mathcal{A}^{\prime}\right)$ ? 
Finally, let us show Theorem 1.13.

Proof of Theorem 1.13. By Proposition 2.6, this is true when $\ell \leq 3$. Hence we may assume that $\ell=4$. By Proposition 2.6 again, whether $\mathcal{A}$ has a filtration $\left\{\mathcal{A}_{i}\right\}_{i=0}^{n}$ such that $\mathcal{A}_{i}$ is all locally free or not depends only on $L(\mathcal{A})$. If there are no such locally free filtrations of $\mathcal{A}$, then $\mathcal{A}$ does not have a free filtration. Assume that there is a locally free filtration. Then it suffices to show that, if $\mathcal{B}:=\mathcal{A} \cup\{H\}$ is locally free, and $\mathcal{A}$ is free, then whether $\mathcal{B}$ is free or not depends only on $L(\mathcal{B})$. Let $\chi_{0}(\mathcal{A} ; t)=(t-a)(t-b)(t-c)$ with $a \leq b \leq c$. By the proof of Theorem 1.9, Theorems 2.12 and 2.13, $\mathcal{B}$ is free if $|\mathcal{A}|-\left|\mathcal{A}^{H}\right| \in\{b, c\}$. Also, if both $\mathcal{A}$ and $\mathcal{B}$ are free, then $|\mathcal{A}|-\left|\mathcal{A}^{H}\right|$ is a root of $\chi(\mathcal{A} ; t)$ by Theorem 2.4. Thus we may assume that $|\mathcal{A}|-\left|\mathcal{A}^{H}\right|=a<b$. Let $\theta_{a}, \theta_{b}, \theta_{c}$ be a basis for $D_{L}(\mathcal{A})$ of corresponding degrees, where $L \in \mathcal{A}$. If $\theta_{a}\left(\alpha_{H}\right) \notin S \alpha_{H}$, then by Proposition 2.3 , we may put

$$
\theta_{a}\left(\alpha_{H}\right)=f_{a} \alpha_{H}+B, \theta_{b}\left(\alpha_{H}\right)=f_{b} \alpha_{H}+g_{b} B, \theta_{c}\left(\alpha_{H}\right)=f_{c} \alpha_{H}+g_{c} B,
$$

where $B \in S_{a}$. Thus replacing $\theta_{b}, \theta_{c}$ by $\theta_{b}-g_{b} \theta_{a}, \theta_{c}-g_{c} \theta_{a}$, we may assume that $\theta_{b}, \theta_{c} \in D(\mathcal{B})$ and thus $\mathcal{B}$ is free with basis $\theta_{E}, \alpha_{H} \theta_{a}, \theta_{b}, \theta_{c}$, which contradicts Theorem 2.4 and $|\mathcal{A}|-\left|\mathcal{A}^{H}\right|=a<b \leq c$. Assume that $\theta_{a} \in D(\mathcal{B})$. If $\theta_{b} \in D(\mathcal{B})$, then the same argument as the above shows that $D(\mathcal{B})$ is free with basis $\theta_{E}, \theta_{a}, \theta_{b}, \alpha_{H} \theta_{c}$. Since the same hold if $\theta_{c} \in D(\mathcal{B})$, we may assume that both are not in $D(\mathcal{B})$. Thus we have a splitting surjection $D_{L}(\mathcal{B}) \rightarrow S \theta_{a}$ as a composition of

$$
D_{L}(\mathcal{B}) \subset D_{L}(\mathcal{A}) \rightarrow S \theta_{a} .
$$

Hence $D_{L}(\mathcal{B})=S \theta_{a} \oplus D_{b, c}$ such that $D_{b, c} \subset S \theta_{b} \oplus S \theta_{c}$. Since $\mathcal{B}$ is locally free, so is $D_{b, c}$. Now by Theorem [1.9, $D_{b, c}$ has a minimal free resolution:

$$
0 \rightarrow S[-d-1] \rightarrow S[-b-1] \oplus S[-c-1] \oplus S[-d] \rightarrow D_{b, c} \rightarrow 0 .
$$

Here $b \leq c \leq d$ and $d$ is the level of $\mathcal{B}$, which contradicts Theorem 3.5 and the local freeness of $\mathcal{B}$. So $\theta_{a} \in D_{L}(\mathcal{B})$ cannot occur.

As a corollary of the proof above, we have the following.

\section{Corollary 4.7}

Let $H \in \mathcal{A}, \mathcal{A}^{\prime}:=\mathcal{A} \backslash\{H\}$ and assume that $\mathcal{A}^{\prime}$ is free with $\exp \left(\mathcal{A}^{\prime}\right)=$ $\left(1, d_{2}, \ldots, d_{\ell}\right)_{\leq}$. If $\mathcal{A}$ is locally free, then $\mathcal{A}$ is free if and only if $|\mathcal{A}|-\left|\mathcal{A}^{H}\right| \in$ $\left\{d_{\ell-2}, d_{\ell-1}, d_{\ell}\right\}$.

\section{Three dimensional case}

In this section, let $\ell=3$, and we prove Theorem 1.11 by dividing the statements and proving each of them. First note the following special facts when 
$\ell=3$.

\section{Proposition 5.1}

Let $\ell=3$. Then every plus-one generated arrangement is strictly plus-one generated.

Proof. Assume that $\mathcal{A}$ is plus-one generated with $\operatorname{POexp}(\mathcal{A})=\left(1, d_{1}, d_{2}\right)_{\leq}$ and level $d$, i.e., there is a minimal set of homogeneous generators $\theta_{E}, \theta_{1}, \theta_{2}, \varphi$ for $D(\mathcal{A})$ such that $\operatorname{deg} \theta_{i}=d_{i}, \operatorname{deg} \varphi=d$, and

$$
f \theta_{E}+f_{1} \theta_{1}+f_{2} \theta_{2}+\alpha \varphi=0 .
$$

Here $f, f_{i} \in S, \alpha \in V^{*}$. It suffices to show that $\alpha \neq 0$. Assume that $\alpha=0$. Subtract $f_{1} \theta_{1}\left(\alpha_{H}\right) \theta_{E} / \alpha_{H}+f_{2} \theta_{2}\left(\alpha_{H}\right) \theta_{E} / \alpha_{H}$ from (5.1) to obtain that

$$
h \theta_{E}+f_{1} \theta_{1}^{\prime}+f_{2} \theta_{2}^{\prime}=0
$$

where $\theta_{i}^{\prime}:=\theta_{i}-\theta_{i}\left(\alpha_{H}\right) \theta_{E} / \alpha_{H}$. Since $\theta_{i}^{\prime}\left(\alpha_{H}\right)=0$, it holds that $h=0$. So we have $f_{1} \theta_{1}^{\prime}=-f_{2} \theta_{2}^{\prime}$. We may assume that $\left(f_{1}, f_{2}\right)=1$, which implies that $\theta:=\theta_{1}^{\prime} / f_{2}=-\theta_{2}^{\prime} / f_{1} \in D_{H}(\mathcal{A})$. Since the resolution is minimal, $\operatorname{deg} f_{i}>0$ for $i=1,2$. Thus $\theta \in D_{H}(\mathcal{A})_{<d_{1}}=(0)$, a contradiction.

\section{Proposition 5.2}

Let $\ell=3$ and $\mathcal{A}$ be plus-one generated with the minimal set of homogeneous generators $\theta_{E}, \theta_{1}, \theta_{2}, \varphi$ for $D(\mathcal{A})$ such that $\operatorname{deg} \theta_{i}=d_{i}, \operatorname{deg} \varphi=d, \theta_{i}, \varphi \in$ $D_{H}(\mathcal{A})$ for some $H \in \mathcal{A}$ and that

$$
f_{1} \theta_{1}+f_{2} \theta_{2}+\alpha \varphi=0
$$

Then $f_{i} \neq 0(i=1,2), \alpha \neq 0$.

Proof. By definition, $D_{H}(\mathcal{A})$ is generated by three derivations of degrees $d_{1} \leq d_{2} \leq d$. By Proposition [5.1, $\alpha \neq 0$. Assume that $f_{1}=0$. Then by the same proof as in Proposition 5.1, there is $\psi \in D_{H}(\mathcal{A})_{<d_{2}}$ such that $\theta_{2}, \varphi \in S_{>0} \psi$. In particular, $D_{H}(\mathcal{A})$ is generated by $\theta_{1}$ and $\psi$, thus $\mathcal{A}$ is free, a contradiction. The same proof works when $f_{2}=0$.

\section{Proposition 5.3}

Let $\ell=3$ and $\mathcal{A}$ be plus-one generated with $\operatorname{PO} \exp (\mathcal{A})=\left(1, d_{1}, d_{2}\right)_{\leq}$and level $d$. Then $d \geq d_{2}$.

Proof. Apply Proposition 5.2 .

Now let us show the deletion version as follows: 


\section{Theorem 5.4}

Let $\ell=3, \mathcal{A} \ni H$ and $\mathcal{A}^{\prime}:=\mathcal{A} \backslash\{H\}$ not free. Then $\mathcal{A}$ is free with exponents $(1, a, b)$ if and only if $\mathcal{A}^{\prime}$ is strictly plus-one generated with $\operatorname{PO} \exp \left(\mathcal{A}^{\prime}\right)=$ $(1, a, b)$ and level $d=\left|\mathcal{A}^{\prime}\right|-\left|\mathcal{A}^{H}\right|$. Note that the equality $d=\left|\mathcal{A}^{\prime}\right|-\left|\mathcal{A}^{H}\right|$ is equivalent to $b_{2}(\mathcal{A})=a b+a+b$.

Proof. The "only if" part is immediate from Theorem 1.4. Let us prove the "if" part. This direction is similar to Proposition 3.4 in [20]. Let $\theta_{E}, \theta_{1}, \theta_{2}, \varphi \in D\left(\mathcal{A}^{\prime}\right)$ generate $D\left(\mathcal{A}^{\prime}\right)$, where $\operatorname{deg} \theta_{1}=a, \operatorname{deg} \theta_{2}=b$ and $\operatorname{deg} \varphi=d$. Since $b_{2}\left(\mathcal{A}^{\prime}\right)=a b+d$ by Proposition 4.1, the deletion-restriction formula implies that $b_{2}(\mathcal{A})=b_{2}\left(\mathcal{A}^{\prime}\right)+\left|\mathcal{A}^{H}\right|=a b+a+b$. Thus $\chi(\mathcal{A} ; t)=$ $(t-1)(t-a)(t-b)$. By Proposition [2.6, it holds that $\left|\mathcal{A}^{H}\right| \leq a+1$ or $\left|\mathcal{A}^{H}\right|=b+1$, and $\mathcal{A}^{\prime}$ is free if $\left|\mathcal{A}^{H}\right|=a+1$ or $b+1$. Thus we may assume that $\left|\mathcal{A}^{H}\right| \leq a \Longleftrightarrow d=\left|\mathcal{A}^{\prime}\right|-\left|\mathcal{A}^{H}\right|=a+b-\left|\mathcal{A}^{H}\right| \geq b$. If $\theta_{2} \notin D(\mathcal{A})$, then $D\left(\mathcal{A}^{\prime}\right) \supsetneq D(\mathcal{A})$ implies that $d=b$ by Proposition 2.3 , and $\varphi \in D(\mathcal{A})$. Thus replacing $\theta_{2}$ by $\varphi$, we may assume that $\theta_{E}, \theta_{1}, \theta_{2} \in D(\mathcal{A})$ and $\varphi \notin D(\mathcal{A})$. Of course $\alpha_{H} \varphi \in D(\mathcal{A})$. Now consider the relation

$$
f \theta_{E}+f_{1} \theta_{1}+f_{2} \theta_{2}+\alpha \varphi=0
$$

at degree $d+1$ coming from the definition of strict plus-one generatedness and Proposition [5.1. Since $\theta_{E}, \theta_{1}, \theta_{2} \in D(\mathcal{A})$, it holds that $\alpha=c \alpha_{H}$ for $c \in \mathbb{K}$. Since $\mathcal{A}^{\prime}$ is strictly plus-one genedated, $c \neq 0$. Thus $\alpha_{H} \varphi \in D(\mathcal{A}) \cap$ $\left\langle\theta_{E}, \theta_{1}, \theta_{2}\right\rangle_{S}$. Since $\operatorname{rank}_{S} D(\mathcal{A})=3$, it holds that $\theta_{E}, \theta_{1}, \theta_{2}$ are $S$-independent by Propositions 4.2 and 5.2, and the sum of their degrees coincide with $|\mathcal{A}|$ by Proposition 4.1. Hence Saito's criterion shows that $\mathcal{A}$ is free.

We can prove the addition-version.

\section{Theorem 5.5}

Let $\mathcal{A}$ be not free, and $H \in \mathcal{A}$. Then $\mathcal{A}^{\prime}:=\mathcal{A} \backslash\{H\}$ is free with exponents $\left(1, d_{1}, d_{2}\right)$ if and only if $\mathcal{A}$ is strictly plus-one generated with exponents $\left(1, d_{1}+\right.$ $\left.1, d_{2}+1\right)$ and level $d=\left|\mathcal{A}^{H}\right|-1$.

Proof. The "only if" part follows from Theorem 1.9. Let us prove the "if" part. Note that $b_{2}(\mathcal{A})=d_{1} d_{2}+d_{1}+d_{2}+1+d$. Let $\theta_{E}, \theta_{1}, \theta_{2}, \varphi$ be the generators for $D(\mathcal{A})$ such that $\operatorname{deg} \theta_{1}=1+d_{1} \leq \operatorname{deg} \theta_{2}=1+d_{2} \leq d=\left|\mathcal{A}^{H}\right|-$ $1=\operatorname{deg} \varphi$. Estimate $\left|\mathcal{A}^{H}\right|=d+1$. By Proposition 4.1, $\chi_{0}(\mathcal{A} \backslash\{H\} ; t)=$ $\left(t-d_{1}\right)\left(t-d_{2}\right)$. By Proposition 2.6, $1+d=\left|\mathcal{A}^{H}\right| \leq d_{1}+1$ or $d_{2}+1 \leq\left|\mathcal{A}^{H}\right|$. The equalities do not hold since they imply the freeness of $\mathcal{A}$ by Proposition 2.6. Hence we may assume that $d<d_{1}$ or $d_{2}<d$. By Proposition 5.3, $d_{2}<d$. Hence $d_{2}+2 \leq d+1=\left|\mathcal{A}^{H}\right|$. So $D\left(\mathcal{A}^{H}\right)$ has a free basis of degrees 1 and $d\left(\geq d_{2}+1\right)$. Note that we have a degree-preserving Euler restriction 
map $\rho: D(\mathcal{A}) \rightarrow D\left(\mathcal{A}^{H}\right)$. Now assume that $d_{2}+2<d+1$. Then $\rho\left(\theta_{i}\right)$ is of the form $\rho\left(g_{i} \theta_{E}\right)$ for some $g_{i} \in S$. By replacing $\theta_{i}$ by $\theta_{i}-g_{i} \theta_{E}$ if necessary, by Proposition 2.1, we may assume that $\alpha_{H}$ divides both $\theta_{1}$ and $\theta_{2}$. Hence $\theta_{E}, \theta_{1} / \alpha_{H}, \theta_{2} / \alpha_{H} \in D\left(\mathcal{A}^{\prime}\right)$. Since they are $S$-independent by Propositions 4.2 and 5.2 , and $|\mathcal{A}|=2+d_{1}+d_{2}, \mathcal{A}^{\prime}$ is free by Saito's criterion.

Next assume that $d_{2}+1=d$. First assume that $d_{1}+1<d_{2}+1=$ $d$. Let $\psi \in D\left(\mathcal{A}^{H}\right)$ be a part of basis of degree $d$. If $\rho(\varphi)=\psi$, then $\theta_{i}^{\prime}:=\theta_{i}-a_{i} \theta_{E}-b_{i} \varphi \in \alpha_{H} D\left(\mathcal{A}^{\prime}\right)$ for some $a_{i}, b_{i} \in S$ by the same proof above. Again by Proposition [5.2, $\theta_{E}, \theta_{1}^{\prime} / \alpha_{H}, \theta_{2}^{\prime} / \alpha_{H}$ is a basis for $D\left(\mathcal{A}^{\prime}\right)$. Next assume that $\rho(\varphi)=\rho\left(a \theta_{E}\right)$ for some $a \in S$. Since $\rho: D(\mathcal{A}) \rightarrow D\left(\mathcal{A}^{H}\right)$ is generically surjective, we may assume that $\rho\left(\theta_{2}\right)=\psi$. By Proposition 5.2 . we may assume that $\theta_{1}^{\prime}$ above, and $\varphi^{\prime}:=\varphi-a \theta_{E}$ is divisible by $\alpha_{H}$. Then $\theta_{E}, \theta_{1} / \alpha_{H}, \varphi^{\prime} / \alpha_{H}$ is a basis for $D\left(\mathcal{A}^{\prime}\right)$.

Now assume that $d_{1}+1=d_{2}+1=d$. By the above argument, replacing $\theta_{1}, \theta_{2}$ and $\varphi$ if necessary, we may assume that $\rho(\varphi)=\psi$. Then the same proof as above completes the proof.

Proof of Theorem 1.11. Combine Theorems 5.4 and 5.5 .

We may pose the following conjecture.

\section{Conjecture 5.6}

The (strictly) plus-one generatedness of $\mathcal{A}$, and its exponents and level depend only on $L(\mathcal{A})$.

We can show the relation with Terao's conjecture.

\section{Corollary 5.7}

Let $\ell=3$. Then Terao's conjecture follows from Conjecture 5.6.

Proof. Assume that Conjecture 5.6 is true. Assume that $\mathcal{A}, \mathcal{B}$ have the isomorphic intersection lattices, and $\mathcal{A}$ is free with $\exp (\mathcal{A})=\left(1, d_{1}, d_{2}\right)$. Let $H \in \mathcal{A}$ and let $L \in \mathcal{B}$ be the corresponding hyperplane to $H \in \mathcal{A}$ under the lattice isomorphism. Theorem 5.4 shows that $\mathcal{A} \backslash\{H\}$ is strictly plus-one generated with exponents $\left(1, d_{1}, d_{2}\right)$ and level $d:=|\mathcal{A}|-\left|\mathcal{A}^{H}\right|-1$. Then by Theorem 5.4 and Conjecture 5.6, $\mathcal{B} \backslash\{L\}$ is strictly plus-one generated with the same exponents and level as $\mathcal{A} \backslash\{H\}$. Since $d=|\mathcal{A}|-\left|\mathcal{A}^{H}\right|-1=$ $|\mathcal{B}|-\left|\mathcal{B}^{L}\right|-1$, Theorem 5.4 confirms that $\mathcal{B}$ is free.

\section{Possible additions to free arrangements}

First let us introduce a definition. 


\section{Definition 6.1}

For an arrangement $\mathcal{A}$, we say that $\mathcal{B}$ is a free addition of $\mathcal{A}$ if $\mathcal{B} \backslash \mathcal{A}=\{H\}$ and $\mathcal{B}$ is free.

By Theorem 1.4, we know that, if $\mathcal{A}$ admits a free addition, then $\mathcal{A}$ has to be either free or strictly plus-one generated. Then it is natural to ask how many free additions are possible. If $\mathcal{A}$ is free, then it is not easy to estimate. But if $\mathcal{A}$ is strictly plus-one generated with high level, then there is the unique free addition.

\section{Theorem 6.2}

Let $\mathcal{A}$ be next to free minus. Thus by Theorem 1.4, $\mathcal{A}$ is strictly plus-one generated with $\operatorname{PO} \exp (\mathcal{A})=\left(d_{1}, \ldots, d_{\ell}\right)$ and level $d$. If $d>d_{i}$ for all $i$, then $\mathcal{A}$ admits only one free addition.

Proof. Since $\mathcal{A}$ is next to free minus, there is a hyperplane $H \notin \mathcal{A}$ such that $\mathcal{B}:=\mathcal{A} \cup\{H\}$ is free. Let $\theta_{1}=\theta_{E}, \theta_{2}, \ldots, \theta_{\ell}$ be a basis for $D(\mathcal{B})$ such that $\operatorname{deg} \theta_{i}=d_{i}$. Then by Theorem 2.7, for $M:=\left(\theta_{i}\left(x_{j}\right)\right)$, it holds that $\operatorname{det} M=Q(\mathcal{B})=\alpha_{H} Q(\mathcal{A})$. By Theorem 1.4, there is $\varphi \in D(\mathcal{A})_{d} \backslash D(\mathcal{B})$ such that $\theta_{1}, \ldots, \theta_{\ell}$ together with $\varphi$ form a minimal set of homogeneous generators for $D(\mathcal{A})$. Let $L \notin \mathcal{A}$ and $\mathcal{A}_{1}:=\mathcal{A} \cup\{L\}$. Assume that $\mathcal{A}_{1}$ is free. Since $\left|\mathcal{A}_{1}\right|=|\mathcal{B}|=|\mathcal{A}|+1$ and $\operatorname{deg} \theta_{i}<\operatorname{deg} \varphi=d$, the homogeneous basis for $D\left(\mathcal{A}_{1}\right)$ can be expressed as $S$-linear combinations of $\theta_{1}, \ldots, \theta_{\ell}$. Since $\operatorname{det} M=\alpha_{H} Q(\mathcal{A})$, it holds that $\mathcal{B}=\mathcal{A}_{1}$.

In the above proof, we used the following easy proposition.

\section{Proposition 6.3}

Assume that $\mathcal{A}$ is strictly plus-one generated with $\operatorname{POexp}(\mathcal{A})=\left(d_{1}, \ldots, d_{\ell}\right)$ and level $d$. Let $L \notin \mathcal{A}$ and $\mathcal{B}:=\mathcal{A} \cup\{L\}$. If $d>d_{i}$ for all $i$, and $\mathcal{B}$ is free, then $\exp (\mathcal{B})=\left(d_{1}, \ldots, d_{\ell}\right)$.

Proof. Immediate by Theorem 1.4.

\section{Proposition 6.4}

Assume that $\mathcal{A}$ is strictly plus-one generated with $\operatorname{POexp}(\mathcal{A})=\left(d_{1}, \ldots, d_{\ell}\right)$ and level $d$. Let $L \notin \mathcal{A}$ and $\mathcal{B}:=\mathcal{A} \cup\{L\}$. If $d>d_{i}$ for all $i$, and $\mathcal{B}$ is free, then $|\mathcal{A}|-\left|\mathcal{B}^{L}\right|=d$.

Proof. Assume that $\mathcal{B}$ is free. Then Proposition 6.3 implies that $\exp (\mathcal{B})=$ $\left(d_{1}, \ldots, d_{\ell}\right)$ and

$$
b_{2}(\mathcal{B})=\sum_{1 \leq i<j \leq \ell} d_{i} d_{j}, b_{2}(\mathcal{A})=\sum_{2 \leq i<j \leq \ell} d_{i} d_{j}+d
$$


where $d_{1}=1$. Thus $\left|\mathcal{B}^{L}\right|=b_{2}(\mathcal{B})-b_{2}(\mathcal{A})=|\mathcal{A}|-d$.

\section{Question 6.5}

Does the converse of Proposition 6.4 hold true? It suffices to check only when the level is contained in exponents.

\section{$7 \quad$ Examples}

We collect several examples of plus-one generated.

\section{Example 7.1}

Let $\mathcal{A}$ be an arrangement in $\mathbb{R}^{3}$ defined by

$$
Q(\mathcal{A})=x y z(x+y+z)=0 .
$$

The algebraic structure of $D(\mathcal{A})$ is well-known, and let us confirm it by using Theorem 1.11. Let $H:=\{x+y+z=0\}$. Since $\mathcal{A}^{\prime}:=\mathcal{A} \backslash\{H\}$ is free with exponents $(1,1,1)$, and $\left|\mathcal{A}^{H}\right|=3$, Theorem 1.11 shows that $\mathcal{A}$ is strictly plus-pne generated with $\operatorname{PO} \exp (\mathcal{A})=(1,2,2)$ and level 2, i.e.,

$$
0 \rightarrow S[-3] \rightarrow S[-1] \oplus S[-2]^{3} \rightarrow D(\mathcal{A}) \rightarrow 0
$$

is a minimal free resolution. This is a nearly free arrangement of planes as in [12].

\section{Example 7.2}

Let $\mathcal{A}$ be defined by

$$
Q(\mathcal{A})=x y z(y-z)\left(x^{2}-y^{2}\right)\left(x^{2}-4 y^{2}\right)=0 .
$$

Let $H:=\{y=0\}$ and $\mathcal{A}^{\prime}:=\mathcal{A} \backslash\{H\} . \mathcal{A}$ is free with $\exp (\mathcal{A})=(1,2,5) . \mathcal{A}^{\prime}$ is the famous example which is not free but $\pi(\mathcal{A})=(1+t)(1+3 t)^{2}$. Since $\left|\mathcal{A}^{\prime}\right|-\left|\mathcal{A}^{H}\right|=5$, Theorem 1.11 implies that $\mathcal{A}^{\prime}$ is strictly plus-one generated with $\operatorname{PO} \exp \left(\mathcal{A}^{\prime}\right)=(1,2,5)$ and level 5, i.e.,

$$
0 \rightarrow S[-6] \rightarrow S[-1] \oplus S[-2] \oplus S[-5]^{2} \rightarrow D\left(\mathcal{A}^{\prime}\right) \rightarrow 0
$$

is a minimal free resolution. This is a nearly free arrangement of planes as in [12].

\section{Example 7.3}

Let $\mathcal{A}$ be defined by

$$
Q(\mathcal{A})=x y z\left(x^{2}-y^{2}\right)\left(x^{2}-z^{2}\right)\left(y^{2}-z^{2}\right)\left((y-x)^{2}-z^{2}\right)\left((y+x)^{2}-z^{2}\right) .
$$


Let $H:=\{y=0\}$ and $L$ be a generic line not in $\mathcal{A}$. $\mathcal{A}$ is free with $\exp (\mathcal{A})=(1,5,7)$. Thus by Theorem 1.11, $\mathcal{A}^{\prime}:=\mathcal{A} \backslash\{H\}$ is is strictly plus-one generated with $\operatorname{PO} \exp \left(\mathcal{A}^{\prime}\right)=(1,5,7)$ and level 8, i.e.,

$$
0 \rightarrow S[-9] \rightarrow S[-1] \oplus S[-5] \oplus S[-7] \oplus S[-8] \rightarrow D\left(\mathcal{A}^{\prime}\right) \rightarrow 0
$$

is a minimal free resolution, so this is not nearly free. Also, by Theorem 6.2. there are no plane $K \neq H$ such that $\mathcal{A}^{\prime} \cup\{K\}$ is free.

For $\mathcal{B}:=\mathcal{A} \cup\{L\}$, by Theorem 1.11, $\mathcal{B}$ is is strictly plus-one generated with $\operatorname{PO} \exp \left(\mathcal{A}^{\prime}\right)=(1,6,8)$ and level 12, i.e.,

$$
0 \rightarrow S[-13] \rightarrow S[-1] \oplus S[-6] \oplus S[-8] \oplus S[-12] \rightarrow D(\mathcal{B}) \rightarrow 0
$$

is a minimal free resolution. This is not nearly free.

\section{Example 7.4}

Let $\mathcal{A}$ be the reflection arrangement corresponding to the complex reflection group $G_{29}$. Then we know that, by [16], Appendix $C, \mathcal{A}$ is free with $\exp (\mathcal{A})=$ $(1,9,13,17)$, and $\mathcal{A}^{H}$ is free with $\exp \left(\mathcal{A}^{H}\right)=(1,9,11)$ for any $H \in \mathcal{A}$. Hence $\mathcal{A}^{\prime}:=\mathcal{A} \backslash\{H\}$ is not free for all $H$, and Theorem 1.4 shows that $\mathcal{A}^{\prime}$ is strictly plus-one generated with $\mathrm{PO} \exp \left(\mathcal{A}^{\prime}\right)=(1,9,13,17)$ and level 18, i.e.,

$$
0 \rightarrow S[-19] \rightarrow S[-1] \oplus S[-9] \oplus S[-13] \oplus S[-17] \oplus S[-18] \rightarrow D\left(\mathcal{A}^{\prime}\right) \rightarrow 0
$$

is a minimal free resolution. Also, by Theorem 6.2, there are no plane $K \neq H$ such that $\mathcal{A}^{\prime} \cup\{K\}$ is free. This is not nearly free.

\section{Example 7.5}

Let $\ell=4$ and $\mathcal{A}$ be defined as

$$
x y z w(x-y)(y-z)(z-w)(x+w)=0
$$

in $V=\mathbb{R}^{4}$, and $H:=\{x+w=0\}$. Then $|\mathcal{A}|=8$ and $\left|\mathcal{A}^{H}\right|=6$. Moreover, $\mathcal{A}^{\prime}:=\mathcal{A} \backslash\{H\}$ is supersolvable, hence free with exponents $(1,2,2,2)$. However, it is easy to check that $\mathcal{A}$ is neither free nor plus-one generated, since $D(\mathcal{A})$ has the projective dimension 2. In fact, since $\left|\mathcal{A}^{\prime}\right|-\left|\mathcal{A}^{H}\right|=1$, the condition in Theorem 1.4 is not satisfied. Moreover, $D(\mathcal{A})$ has a minimal set of generators consisting of the Euler derivation, and 6-derivations of degree 3 .

\section{Application to the deletion theorem}

In [4], the deletion theorem is proved to be combinatorial, i.e., the following is shown. 
Theorem 8.1 ([4], Theorem 1.2)

Let $\mathcal{A}$ be free and $H \in \mathcal{A}$. Then $\mathcal{A}^{\prime}:=\mathcal{A} \backslash\{H\}$ is free if and only if $\pi\left(\mathcal{A}_{X}^{H} ; t\right) \mid \pi\left(\mathcal{A}_{X} ; t\right)$ for all $X \in L\left(\mathcal{A}^{H}\right)$. Equivalently, $\mathcal{A}^{\prime}$ is free if and only if for all $X \in L\left(\mathcal{A}^{H}\right)$, it holds that

$$
b_{2}\left(\mathcal{A}_{X}\right)=b_{2}\left(\mathcal{A}_{X}^{H}\right)+\left|\mathcal{A}_{X}^{H}\right|\left(\left|\mathcal{A}_{X}\right|-\left|\mathcal{A}_{X}^{H}\right|\right),
$$

and $-\left(\left|\mathcal{A}_{X}\right|-\left|\mathcal{A}_{X}^{H}\right|\right)^{-1}$ is a root of $\pi\left(\mathcal{A}_{X} ; t\right)$.

In the proof of Theorem 8.1 , the algebraic structure of $D\left(\mathcal{A}^{H}\right)$ played the key role. By using the theory on the structure of $D\left(\mathcal{A}^{\prime}\right)$, we can show that Theorem 8.1 can be expressed only in terms of the number of hyperplanes.

\section{Theorem 8.2}

Let $\mathcal{A}$ be free and $H \in \mathcal{A}$. Then $\mathcal{A}^{\prime}:=\mathcal{A} \backslash\{H\}$ is free if and only if $s_{X}:=-\left(\left|\mathcal{A}_{X}\right|-\left|\mathcal{A}_{X}^{H}\right|\right)^{-1}$ is a non-zero root of $\pi\left(\mathcal{A}_{X} ; t\right)$ for all $X \in L\left(\mathcal{A}^{H}\right)$.

Proof. Induction on $\ell \geq 2$. When $\ell=2$ there is nothing to show, When $\ell=3$, it follows by Proposition 2.6. Assume that $\ell \geq 4$. Then by the induction hypothesis, we may assume that $\mathcal{A}^{\prime}$ is locally free. By Theorem 1.4, $\mathcal{A}^{\prime}$ is either free or strictly plus-one generated, and the latter cannot occur by Theorem 3.5 .

A corollary of the proof of Theorem 8.2 is as follows:

\section{Corollary 8.3}

Let $\mathcal{A}$ be free and $H \in \mathcal{A}$. Assume that $\mathcal{A}^{\prime}$ is locally free. Then $\mathcal{A}^{\prime}:=\mathcal{A} \backslash\{H\}$ is free if and only if $-\left(|\mathcal{A}|-\left|\mathcal{A}^{H}\right|\right)^{-1}$ is a non-zero root of $\pi(\mathcal{A} ; t)$.

\section{References}

[1] T. Abe, Roots of characteristic polynomials and and intersection points of line arrangements. J. Singularities, 8 (2014), 100-117.

[2] T. Abe, Divisionally free arrangements of hyperplanes. Invent. Math. 204 (2016), no. 1, 317-346.

[3] T. Abe, Restrictions of free arrangements and the division theorem. Proceedings of the Intensive Period "Perspectives in Lie Theory", Springer INdAM Series ; 19 (2017), 389-401.

[4] T. Abe, Deletion theorem and combinatorics of hyperplane arrangements. Math. Ann., DOI: https://doi.org/10.1007/s00208018-1713-9 (online). 
[5] T. Abe, M. Barakat, M. Cuntz, T. Hoge, H. Terao, The freeness of ideal subarrangements of Weyl arrangements. arXiv:1304.8033. J. Eur. Math. Soc., 18 (2016). no. 6, 1339-1348.

[6] T. Abe and H. Terao, Free filtrations of affine Weyl arrangements and the ideal-Shi arrangements. J. Alg. Combin., 43 (2016), no. $133-44$.

[7] T. Abe and H. Terao, Multiple addition, deletion and restriction theorems for hyperplane arrangements. arXiv:1801.01790

[8] T. Abe, H. Terao and M. Wakefield, The characteristic polynomial of a multiarrangement. Adv. in Math., 215 (2007), 825-838.

[9] T. Abe, H. Terao and M. Wakefield, The Euler multiplicity and addition-deletion theorems for multiarrangements. J. London Math. Soc., 77 (2008), no. 2, 335-348.

[10] T. Abe and M. Yoshinaga, Free arrangements and coefficients of characteristic polynomials. Math. Z., 275 (2013), Issue 3, 911-919.

[11] G. Denham and M. Schulze, Complexes, duality and Chern classes of logarithmic forms along hyperplane arrangements. Arrangements of hyperplanesSapporo 2009, 27-57, Adv. Stud. Pure Math., 62, Math. Soc. Japan, Tokyo, 2012.

[12] A. Dimca and G, Sticlaru, Free and nearly free curves vs. rational cuspidal plane curves. Publ. RIMS, Kyoto Univ. 54 (2018), 163179 .

[13] A. Dimca and G, Sticlaru, Free and nearly free surfaces in $\mathbf{P}^{3}$. arXiv:1507.03450.

[14] P. H. Edelman and V. Reiner, A counterexample to Orlik's conjecture. Proc. Amer. Math. Soc., 118 (1993), 927-929.

[15] T. Hoge, a private communication. 2013.

[16] P. Orlik and H. Terao, Arrangements of hyperplanes. Grundlehren der Mathematischen Wissenschaften, 300. Springer-Verlag, Berlin, 1992.

[17] K. Saito, Theory of logarithmic differential forms and logarithmic vector fields. J. Fac. Sci. Univ. Tokyo 27 (1980), 265-291. 
[18] H. Terao, Arrangements of hyperplanes and their freeness I, II. J. Fac. Sci. Univ. Tokyo 27 (1980), 293-320.

[19] H. Terao, Generalized exponents of a free arrangement of hyperplanes and Shephard-Todd-Brieskorn formula. Invent. math. 63 (1981), 159-179.

[20] M. Wakefield, Derivation degree sequences of non-free arrangements. arXiv:1807.07613.

[21] M. Yoshinaga, Characterization of a free arrangement and conjecture of Edelman and Reiner. Invent. Math. 157 (2004), no. 2, 449-454.

[22] M. Yoshinaga, On the freeness of 3-arrangements. Bull. London Math. Soc. 37 (2005), no. 1, 126-134.

[23] M. Yoshinaga, Freeness of hyperplane arrangements and related topics. Annales de la Faculte des Sciences de Toulouse, 23 (2014), no. $2,483-512$.

[24] G. M. Ziegler, Multiarrangements of hyperplanes and their freeness. Singularities (Iowa City, IA, 1986), 345-359, Contemp. Math., 90, Amer. Math. Soc., Providence, RI, 1989. 